\title{
Pertinence d'une sélection des pesticides à mesurer dans un réseau de surveillance de la qualité des eaux superficielles à l'aide d'une méthode simplifiée d'évaluation des risques Relevance of the Selection of Pesticides for a River Monitoring Network with a Simple Risk Assessment Method
}

\section{Babut et C. Breuzin}

Volume 13, numéro 4, 2000

URI : https://id.erudit.org/iderudit/705398ar

DOI : https://doi.org/10.7202/705398ar

\section{Aller au sommaire du numéro}

\section{Éditeur(s)}

Université du Québec - INRS-Eau, Terre et Environnement (INRS-ETE)

ISSN

0992-7158 (imprimé)

1718-8598 (numérique)

\section{Découvrir la revue}

Citer cet article

Babut, M. \& Breuzin, C. (2000). Pertinence d'une sélection des pesticides à mesurer dans un réseau de surveillance de la qualité des eaux superficielles à l'aide d'une méthode simplifiée d'évaluation des risques. Revue des sciences de l'eau / Journal of Water Science, 13(4), 363-378. https://doi.org/10.7202/705398ar

\section{Résumé de l'article}

L'une des difficultés rencontrées couramment dans la conception des réseaux de mesure - au moins en ce qui concerne les micropolluants - porte sur la sélection des paramètres à mesurer. C'est notamment le cas pour les pesticides, dont plusieurs centaines sont utilisées en agriculture, mais qu'il est impossible de surveiller dans les eaux en totalité pour des raisons à la fois techniques et économiques. C'est la raison pour laquelle les autorités françaises ont fait procéder à la mise au point d'une méthode de sélection des matières actives utilisées en agriculture basée sur l'évaluation du risque. Dans cette méthode, l'exposition est figurée par un rang combinant les données relatives aux usages des matières actives (superficie, dose par ha) et leurs caractéristiques physico-chimiques. Le danger est représenté par la toxicité, soit pour l'homme, soit pour les espèces aquatiques. Cette approche a été appliquée à l'échelle nationale et dans un certain nombre de régions françaises, dont l'Alsace et la Lorraine. Les résultats des mesures de pesticides réalisées ensuite pendant un an ont été confrontés aux indices d'exposition obtenus. Les substances détectées le plus fréquemment correspondent effectivement à celles dotées des rangs d'exposition les plus élevés (ajustement exponentiel, $\mathrm{r}^{2} \approx 0.82$ ); cependant, le diuron apparaît à une fréquence plus élevée que celle attendue, en raison de ses usages non agricoles. La corrélation est moins bonne pour les substances dont les rangs d'exposition sont proches de la valeur considérée comme significative pour les eaux superficielles, ce qui peut provenir soit de l'utilisation de données erronées lors de la sélection, soit d'un poids insuffisant attribué à certains facteurs dans la méthode de sélection, soit enfin d'aléas météorologiques. 


\title{
Pertinence d'une sélection des pesticides à mesurer dans un réseau de surveillance de la qualité des eaux superficielles à l'aide d'une méthode simplifiée d'évaluation des risques
}

\author{
Relevance of the selection of pesticides for a river \\ monitoring network with a simple risk assessment \\ method
}

M. BABUT ${ }^{1 *}$, C. BREUZIN ${ }^{2}$

Reçu le 21 mars 1999, accepté le 2 novembre 2000**.

\section{SUMMARY}

\begin{abstract}
Monitoring of micropollutants is a rather recent activity (10-15 years), at least in surface waters; because of the need for sophisticated analytical methods and of the potential number of analytes, this type of activity is confronted with important economic constraints, which require that one make a selection among the range of substances to monitor. Among organic micropollutants, pesticides constitute a well-identified category, since they are used mainly in agriculture; this use on broad surfaces may have important impacts on the quality of surface water. Various methods have been used to select those pesticides likely to have the greatest impacts on water quality; some of these methods might be considered to be "hazard assessment", whereas others correspond to simplified "risk assessment" methods (this appears particularly true for pesticides, of which several hundreds are used in agriculture). Recently, a French panel of experts mandated by different Ministries designed a selection method called SIRIS, which allows one to define three different lists of pesticides according to the media to be monitored (surface or ground-water) and to the monitoring objectives (ecosystem protection, drinking water production). This paper deals with the application of the SIRIS method at a regional level, in the context of a permanent survey of river quality.
\end{abstract}

As a simplified risk assessment method, SIRIS combines data on hazard and exposure; hazard is estimated by a single parameter, either toxicity for aquatic species or acceptable daily intake (ADI). Exposure represents the probability that a transfer to water bodies may occur; for surface water, this probability is influenced by the crop acreage, the applied dose $(\mathrm{kg} / \mathrm{ha})$, the solubility, the pesticide half-life, the hydrolysis and the distribution coefficient between water and organic matter (Koc). These factors are considered

1. Cemagref, Groupement de Lyon, 3 bis quai Chauveau, 69336 Lyon cedex 09, France.

2. Agence de l'eau Rhin-Meuse, BP 30019, 57161 Moulins-les-Metz, France.

* Correspondance. E-mail : marc.babut@cemagref.fr

** Les commentaires seront reçus jusqu'au 14 septembre 2001. 
in this hierarchical order, and for each substance a score is assigned to each of these factors among three possible values " " 0 " = slight, " $\mathrm{m}$ " = medium, "d" = high, according to the relative influence on transfer); finally exposure is estimated by a relative rank obtained by a combination of these values following a "penalisation" principle. Two tables are available for applying this approach at a regional level: the first contains the values $(0, m, d)$ assigned to more than 300 substances by the expert panel for solubility, half-life, etc., and should be completed with crop acreage and dose. The second table provides the ranks corresponding to the different combinations of $o, m, d$ values. A final rank of 35 was considered by consensus to be a pragmatic threshold for the transfer to surface water. This method was applied in 1996 in two regions in France (Alsace and Lorraine) separately; most of the selected chemicals (but unfortunately not all, due to technical constraints) were then analysed monthly in surface waters ( 24 sampling points, yielding 144 samples in Alsace and 169 in Lorraine). Occurrences fell between $0 \%$ and $60 \%$ in Alsace, and between $0 \%$ and $90 \%$ in Lorraine; in both regions, the most frequently detected chemicals were atrazine and diuron.

The relevance of the selection method may be discussed under several aspects: the choice of the factors, their order, the position of thresholds corresponding to $\mathrm{o}, \mathrm{m}, \mathrm{d}$ values, the value of the overall threshold, and the availability of the data. Some pesticides are not ranked only because no data were available concerning their solubility, hydrolysis rate or Koc, but the relative importance of such gaps cannot be appreciated with the current set of data. Other items may be assessed through the comparison of the exposure rank versus the occurrence. This relationship takes an exponential shape, with some anomalies: for example, the occurrence of diuron in Alsace is higher than expected, based on its exposure rank. This situation can be explained by the fact that there are non-agricultural uses of this substance, such that the exposure rank appears to be underestimated. For other substances, like aldicarb and chlorpyrifos-ethyl, discrepancies are observed between the exposure rank and occurrences, when comparing with substances with higher exposure ranks. This anomaly may be due to poor data quality. For carbendazime, the occurrence in Lorraine appears underestimated, probably because of a dry period deficit after the application. Finally, chlortoluron received the same rank in the 2 regions, but is more frequently detected in Lorraine; crop acreage may have been overestimated in Alsace. However, the dataset is still limited to one year of sampling; some discrepancies may appear less important when more data are available. For chemicals with ranks $>50$, there is a good exponential fit between ranks and occurrences $\left(y=0.0235 \% e^{0.0739 x} ; r^{2}=0.82\right)$. This observation means that pesticides with ranks $>\mathbf{5 0}$ are systematically encountered in surface waters; however, the current threshold (35) should be maintained, because some substances with ranks $<50$ are also detected. Thus, the SIRIS method appears to be a good tool for selecting agricultural pesticides for monitoring purposes at a regional level.

Key-words: pesticide, carbendazime, herbicide, triazine, atrazine, diuron, selection model, risk index.

\section{RÉSUMÉ}

L'une des difficultés rencontrées couramment dans la conception des réseaux de mesure - au moins en ce qui concerne les micropolluants - porte sur la sélection des paramètres à mesurer. C'est notamment le cas pour les pesticides, dont plusieurs centaines sont utilisées en agriculture, mais qu'il est impossible de surveiller dans les eaux en totalité pour des raisons à la fois techniques et économiques. C'est la raison pour laquelle les autorités françaises ont fait procéder à la mise au point d'une méthode de sélection des 
matières actives utilisées en agriculture basée sur l'évaluation du risque. Dans cette méthode, l'exposition est figurée par un rang combinant les données relatives aux usages des matières actives (superficie, dose par ha) et leurs caractéristiques physicochimiques. Le danger est représenté par la toxicité, soit pour l'homme, soit pour les espèces aquatiques. Cette approche a été appliquée à l'échelle nationale et dans un certain nombre de régions françaises, dont l'Alsace et la Lorraine. Les résultats des mesures de pesticides réalisées ensuite pendant un an ont été confrontés aux indices d'exposition obtenus. Les substances détectées le plus fréquemment correspondent effectivement à celles dotées des rangs d'exposition les plus élevés (ajustement exponentiel, $r^{2} \approx 0.82$ ) ; cependant, le diuron apparaît à une fréquence plus élevée que celle attendue, en raison de ses usages non agricoles. La corrélation est moins bonne pour les substances dont les rangs d'exposition sont proches de la valeur considérée comme significative pour les eaux superficielles, ce qui peut provenir soit de l'utilisation de données erronées lors de la sélection, soit d'un poids insuffisant attribué à certains facteurs dans la méthode de sélection, soit enfin d'aléas météorologiques.

Mots clés : pesticide, carbendazime, herbicide, triazine, atrazine, diuron, sélection, modèle, risque, indice.

\section{1 - INTRODUCTION}

La prise en compte des micropolluants dans les réseaux de surveillance de la qualité des eaux, notamment superficielles, est relativement récente (10 à 15 ans). Recourant à des techniques d'analyse sophistiquées, ce type de surveillance s'est trouvé confronté à des contraintes économiques importantes, rendues plus aiguës par la multiplicité des paramètres susceptibles de devoir être mesurés. Cette multiplicité rend à l'évidence nécessaire une sélection rationnelle des substances à surveiller (UN/ECE, 1996). Parmi les micropolluants, les pesticides constituent une famille relativement bien délimitée - par l'usage majoritairement agricole qui en est fait --, qui intéresse fortement la qualité des eaux de par son utilisation même, et qui présente en même temps une grande diversité de propriétés physicochimiques. Plusieurs méthodes ont été proposées dans différents contextes pour classer les pesticides pertinents vis-à-vis de la qualité de l'eau (RAO et al., 1985 ; JURY et al., 1987). Ces méthodes s'apparentent à une évaluation du danger, dans la mesure où les critères de classement portent sur les caractéristiques des substances, en particulier leur aptitude à l'adsorption et leur biodégradabilité ; plus rarement, la sélection procède d'une évaluation simplifiée des risques, où l'exposition est figurée par les quantités utilisées et l'occurrence dans les milieux aquatiques (BEEK et BROSELISKE, 1994). D'autres méthodes, basées sur des critères analogues, visent plutôt à classer les matières actives en fonction de leur impact de façon à optimiser les choix des utilisateurs (HORNSBY, 1992 ; NEWMAN, 1995), ou à fonder des décisions d'homologations pour des secteurs particulièrement sensibles (BRITT et al., 1992). Ces méthodes se rapprochent d'une évaluation de risque, dans la mesure où elles tiennent compte de certains effets toxiques sur l'homme, ou sur l'écosystème cible.

Récemment, les ministères français de la Santé, de l'Environnement et de l'Agriculture ont chargé un comité d'experts de mettre au point une méthode 
de sélection, appelée SIRIS (pour Système d'Intégration des Risques par Interaction de Scores) applicable aux eaux souterraines et superficielles, et distinguant la production d'eau potable et la protection des écosystèmes aquatiques (JOUANY et DABENE, 1994). Cette méthode a tout d'abord été mise au point et appliquée à l'échelle nationale, donnant lieu à la publication de trois listes de composés " prioritaires " (deux pour l'usage production d'eau potable, en distinguant eaux souterraines et eaux superficielles, une pour la protection des écosystèmes aquatiques d'eau douce). Dans un deuxième temps, cette méthode a été déclinée dans différentes régions, de façon à prendre en compte la diversité de l'agriculture ; des listes (trois par région) ont ainsi été proposées dans les régions Alsace et Lorraine, qui ont servi de base au programme de surveillance des produits phytosanitaires dans les eaux superficielles en 1997. L'objectif de cet article est de discuter les résultats obtenus pour les eaux superficielles dans ces deux régions, et de tenter de vérifier la pertinence de la méthode de sélection.

\section{2 - MATÉRIEL ET MÉTHODES}

\subsection{Méthode de sélection des paramètres à mesurer}

La méthode de sélection combine d'une part une évaluation d'un paramètre d'exposition, qui décrit l'ensemble des facteurs influençant le transfert vers les milieux aquatiques, et d'autre part une description du danger, représentée soit par la dose journalière admissible (DJA) pour la production d'eau potable, soit par la toxicité aiguë pour des organismes aquatiques. Les données utilisées sont issues de la bibliographie, ou parfois des dossiers d'homologation ; les quantités appliquées et les superficies développées peuvent provenir d'enquêtes statistiques, voire d'estimations théoriques.

Tableau 1 Principe de pénalisation.

Table 1 Penalty method.

\begin{tabular}{|c|c|c|}
\hline Facteur 1 & Facteur 2 & Rang \\
\hline \multirow{3}{*}{$0=0$} & $0=0$ & $0+0=0$ \\
& $m=1$ & $0+1=1$ \\
& $d=2$ & $0+2=2$ \\
$m=2$ & $0=0$ & $2+0=2$ \\
& $m=1$ & $2+1=3$ \\
& $d=3$ & $2+3=5$ \\
$d=5$ & $0=0$ & $5+0=5$ \\
& $m=2$ & $5+2=7$ \\
& $d=4$ & $5+4=9$ \\
\hline
\end{tabular}


Le paramètre d'exposition exprime par une donnée unique (rang) la probabilité de transfert vers les eaux ; dans le cas des eaux superficielles, les facteurs déterminants sont, dans l'ordre, la superficie de la culture, la dose de matière active à l'hectare, la solubilité, la demi-vie, l'aptitude à l'hydrolyse et le coefficient de partage eau/matière organique (Koc). Pour chacun de ces facteurs, on détermine trois classes, notées « 0 », « $m$ » et « $d$ », du plus favorable (valeur du facteur entrainant le moins de transfert) au plus défavorable. La combinaison de ces valeurs détermine un rang par application d'un principe d'auto-pénalisation, dont le tableau 1 résume le principe pour deux facteurs. L'interaction de deux facteurs défavorables engendre un classement (rang) également défavorable ; d'autre part, un item pour lequel le premier facteur est défavorable ne peut être in fine mieux classé qu'un autre item pour lequel ce facteur est plus favorable, quelle que soit la valeur prise par les autres facteurs pris en compte par la suite.

Le document (JOUANY et DABENE, 1994) fournit un tableau complet des pénalités pour près de 300 matières actives ; ce tableau peut également être obtenu sous forme d'une feuille de calcul. Lors de l'application à des échelles régionales, le tableau doit être complété pour les facteurs relatifs aux superficies développées et aux doses d'emploi (Comité de liaison "eau-produits antiparasitaires "1995), lorsque ces dernières sont différentes des doses homologuées. La valeur des Koc dépendant du type de sol et de sa teneur en matière organique, il conviendrait en principe de les ajuster également lors de l'application de la méthode de sélection à l'échelle régionale, mais dans la pratique on s'aperçoit que cela n'est possible que pour quelques substances.

La pertinence du classement obtenu dépend de deux critères, qui sont d'une part l'obtention d'un consensus entre experts de différentes origines professionnelles pour la définition de l'ordre des facteurs et des bornes de classes, et d'autre part la qualité des données utilisées, notamment en ce qui concerne les superficies développées et les doses appliquées, qui peuvent être inférieures aux doses homologuées.

Lors de l'application de cette méthode en Lorraine, les données pour les superficies et les doses d'usage ont été tirées d'enquêtes statistiques (CETIOM, 1995), tandis qu'en Alsace les superficies et les doses d'emploi ont été estimées directement par les services du ministère de l'Agriculture.

\subsection{Implantation des points de prélèvement}

Les points de prélèvement font partie du "Réseau National de Bassin » (RNB), qui a pour vocation de fournir les données nécessaires à l'appréciation de la qualité des eaux superficielles ; ils ont été choisis en tenant compte de l'occupation des sols (SIEE et SRPV Midi-Pyrénées, 1997), et placés soit dans des zones intermédiaires du réseau hydrographique, soit à l'aval de grands bassins versants. La localisation de ces points figure en annexe. L'échantillonnage est réalisé de manière ponctuelle, à un rythme mensuel pour les stations situées dans les zones intermédiaires, et toutes les 4 semaines ( 13 fois par an) pour les stations situées à l'aval des grands bassins versants. Ce programme est résumé dans le tableau 2. Les prélèvements ont été divisés en tournée, chaque tournée correspondant aux stations prélevées dans la même journée. À chaque fin de journée les échantillons sont transmis aux laboratoires, soit directement par les agents préleveurs, soit par transporteur. Tous les échantillons sont conservés à une température de $4^{\circ} \mathrm{C}$ à l'abri de la lumière. 
Tableau 2 Résumé du programme annuel d'échantillonnage.

Table 2 Summary of the yearly sampling program.

\begin{tabular}{|l|c|c|c|}
\hline Type de stations de prélèvement & $\begin{array}{c}\text { Nombre } \\
\text { d'échantillons } \\
\text { par an }\end{array}$ & $\begin{array}{c}\text { Nombre } \\
\text { de stations } \\
\text { en Alsace }\end{array}$ & $\begin{array}{c}\text { Nombre } \\
\text { de stations } \\
\text { en Lorraine }\end{array}$ \\
\hline Évaluation (zones intermédiaires) & 12 & 11 & 12 \\
Bilan (secteurs aval de grands bassins) & 13 & 1 & 3 \\
Nombre de mesures réalisées & 144 & 169 \\
\hline
\end{tabular}

\subsection{Méthodes d'analyse}

La mise en œuvre des prélèvements et analyses de pesticides dans le cadre du RNB est répartie en plusieurs lots ; les substances sont analysées par groupes, dans les conditions résumées au tableau 3. L'ensemble des laboratoires d'analyses ayant pratiqué les déterminations sont accrédités par le Comité Français d'Accréditation (COFRAC ; programme 100 " Analyses des eaux "). Ce programme d'accréditation impose notamment d'utiliser des méthodes validées, dont les rendements d'extraction sont donc connus. Ces rendements sont déterminés par addition de quantités précises des pesticides recherchés à des eaux naturelles. Dans le cas des analyses présentées ici, ils sont en général compris entre $70 \%$ et $101 \%$.

Dans l'attente de la phase d'extraction les échantillons sont conservés en chambre froide, à l'abri de la lumière. Les substances à recherchées sont extraites dans un délai allant de $24 \mathrm{~h}$ à 7 jours maximum après le prélèvements. Ces délais sont établis en fonction des normes en vigueur et des connaissances particulières acquises sur ces substances. Les extraits plus stables sont conservés en chambre froide, à l'abri de la lumière et analysés dans les 3 semaines. Les résultats supérieurs aux limites de quantification, sont confirmés soit par MS/MS, soit par une analyse sur une nouvelle colonne, soit encore par une extraction par un autre solvant ou par une analyse plus fine des spectres obtenus.

\section{3 - RÉSULTATS}

\subsection{Listes de pesticides à mesurer dans les eaux superficielles}

Deux listes ont donc été établies dans chacune des deux régions :

- liste A : Substances à rechercher dans les eaux superficielles : protection des consommateurs ;

- liste $B$ : Substances à rechercher dans les eaux superficielles : pertinence vis-à-vis de l'écosystème. 
Tableau 3 Méthodes d'analyse appliquées.

Table 3 Analytical methods.

\begin{tabular}{|c|c|c|c|}
\hline Lot & Laboratoire & Groupe & Méthode \\
\hline \multirow[t]{4}{*}{$\begin{array}{l}\text { Alsace, stations } \\
\text { d'évaluation }\end{array}$} & \multirow[t]{4}{*}{$\begin{array}{l}\text { Centre d'Analyses } \\
\text { et de Recherches, } \\
\text { Strasbourg }\end{array}$} & triazines, phényl-urées & $\begin{array}{l}\text { NF EN ISO } 11369 \\
\text { Extraction : Liq./Sol. } \\
\text { Détection : HPLC/DAD }\end{array}$ \\
\hline & & $\begin{array}{l}\text { organo-phosphorés, } \\
\text { pendiméthaline, } \\
\text { trifluraline, deltaméthrine }\end{array}$ & $\begin{array}{c}\text { Extraction: } \\
\text { Liq./Sol. (hexane) } \\
\text { Détection : GC/MS }\end{array}$ \\
\hline & & $\mathrm{N}$-méthyl carbamates & $\begin{array}{l}\text { Extraction : Liq./Sol. } \\
\text { Détection : } \\
\text { HPLC dérivatisation } \\
\text { post colonne, } \\
\text { fluorimétrie }\end{array}$ \\
\hline & & $\mathrm{N}$-aryl carbamates & $\begin{array}{l}\text { Extraction : Liq./Sol. } \\
\text { Détection : HPLC/DAD }\end{array}$ \\
\hline \multirow[t]{2}{*}{$\begin{array}{l}\text { Lorraine, stations } \\
\text { d'évaluation }\end{array}$} & \multirow[t]{2}{*}{ Lab. Wolff } & $\begin{array}{c}\text { atrazine, deltaméthrine, } \\
\text { lambda cyhalothrine, } \\
\text { trifluraline }\end{array}$ & $\begin{array}{c}\text { Extraction : } \\
\text { Liq./Liq. (CH2Cl2) } \\
\text { Détection : GC/MS }\end{array}$ \\
\hline & & $\begin{array}{l}\text { carbendazime, } \\
\text { phényl-urées }\end{array}$ & $\begin{array}{l}\text { Extraction : Liq./Sol. } \\
\text { Détection : HPLC/DAD }\end{array}$ \\
\hline \multirow[t]{6}{*}{ Stations bilan } & \multirow[t]{6}{*}{ IRH-Environnement } & triazines & $\begin{array}{c}\text { Extraction : } \\
\text { Liq./Liq. (CH2Cl2) } \\
\text { Détection : GC/TSD }\end{array}$ \\
\hline & & phényl-urées & $\begin{array}{c}\text { Extraction : Liq./Liq. } \\
\text { (Hexane/CH2Cl2) } \\
\text { Détection : UV/DAD }\end{array}$ \\
\hline & & organophosphoré & $\begin{array}{l}\text { Extraction : Liq./Liq. } \\
\text { (CH2Cl2/hexane) } \\
\text { Détection : GC/TSD }\end{array}$ \\
\hline & & carbamates & $\begin{array}{l}\text { Extraction : Liq./Sol. } \\
\text { Détection : HPLC/DAD }\end{array}$ \\
\hline & & organochlorés & $\begin{array}{l}\text { Extraction : Liq./Liq. } \\
\text { (CH2Cl2/hexane) } \\
\text { Détection : GC/ECD }\end{array}$ \\
\hline & & thiocarbamates & $\begin{array}{l}\text { Extraction : Liq./Liq. } \\
\text { (hexane, CH2Cl2) } \\
\text { Détection : GC/MS }\end{array}$ \\
\hline
\end{tabular}

Ces deux listes sont établies à partir d'une évaluation commune de l'exposition ; elles diffèrent au niveau des critères d'effet utilisés - DJA pour l'eau potable, toxicité aiguë sur daphnie ou poisson pour l'écosystème - Les listes obtenues figurent au tableau 4.

\subsection{Mesures réalisées en 1997}

Pour des raisons techniques (fiabilité des méthodes d'analyse, limites de dosage trop élevées), certaines des substances listées n'ont pu être mesurées. D'autres en revanche, comme le diuron en Lorraine, ont pu être mesurées malgré leur absence des listes, parce que la méthode d'analyse des phényl-urées 
Tableau 4 Listes des substances à rechercher.

Table 4 Lists of monitored substances.

\begin{tabular}{|c|c|c|c|c|c|}
\hline Consommation & Ecosystème & Rang & Consommation & Ecosystème & Rang \\
\hline \multicolumn{2}{|c|}{ atrazine } & 105 & \multicolumn{2}{|l|}{ alachlore } & \\
\hline & carbendazime & 47 & \multicolumn{2}{|c|}{ aldicarbe } & 38 \\
\hline & chlortoluron & 85 & & & \\
\hline & cyproconazole & & & alphaméthrine & \\
\hline & deltaméthrine & 42 & aminotriazole & & \\
\hline \multicolumn{2}{|c|}{ epoxiconazole } & & \multicolumn{2}{|c|}{ atrazine } & 105 \\
\hline & fenbuconazole & & & captane & \\
\hline & fenpropidine & & & carbendazime & 47 \\
\hline fenpropimorphe & & & \multicolumn{2}{|c|}{ chlorpyriphos-éthyl } & 36 \\
\hline flusilazole & & & & chlortoluron & 85 \\
\hline hexaconazole & & & cyanazine & & \\
\hline ioxynil & & & & deltaméthrine & 42 \\
\hline \multirow[t]{2}{*}{ isoproturon } & & 96 & \multicolumn{2}{|c|}{ dicofol } & \\
\hline & $\lambda$-cyhalothrine & 36 & diqual & & \\
\hline \multicolumn{2}{|c|}{ rHCH (lindane) } & 49 & \multicolumn{2}{|c|}{ diuron } & 55 \\
\hline \multicolumn{2}{|c|}{ linuron } & 37 & \multicolumn{2}{|c|}{ epoxiconazole } & \\
\hline prosulfocarbe & & 51 & & fenpropidine & \\
\hline triallate & & & fenpropimorphe & & \\
\hline \multicolumn{2}{|c|}{ trifluraline } & 58 & flufenoxuron & & \\
\hline \multirow[t]{14}{*}{ A- Lorraine } & & & flusilazole & & \\
\hline & & & \multicolumn{2}{|c|}{ folpel } & \\
\hline & & & \multicolumn{2}{|c|}{ y $\mathrm{HCH}$ (lindane) } & 66 \\
\hline & & & isophenphos & & \\
\hline & & & isoproturon & & 96 \\
\hline & & & & $\lambda$-cyhalothrine & 36 \\
\hline & & & & méthomyl & \\
\hline & & & \multicolumn{2}{|c|}{ paraquat } & \\
\hline & & & \multicolumn{2}{|c|}{ parathion-méthyl } & 38 \\
\hline & & & & pendiméthaline & 74 \\
\hline & & & simazine & & 65 \\
\hline & & & terbuthylazine & & \\
\hline & & & & thiodicarbe & 43 \\
\hline & & & & & 74 \\
\hline
\end{tabular}

B- Alsace 
permettait de le faire sans modification du coût. Les résultats obtenus après une année de mesures, présentés sous forme de fréquences de détection, sont résumés au tableau 5. Les fréquences de détection ont été calculées sur l'ensemble des points de prélèvement. En Alsace, les fréquences vont de $0 \%$ à environ $60 \%$, sur 144 mesures, tandis qu'en Lorraine les fréquences vont de $0 \%$ à $90 \%$ sur 169 mesures. Dans les deux régions, ce sont l'atrazine et le diuron qui sont le plus fréquemment détectés ; en Alsace, les substances suivantes dans l'ordre décroissant des fréquences sont à moins de $10 \%$, à l'exception du chlortoluron (11,8\%) ; en Lorraine, après l'atrazine et le diuron, on trouve 2 phényl-urées entre 20 et $30 \%$, les autres substances étant inférieures à $3 \%$ des détections. Malgré la dimension importante des bassins versants, ces fréquences sont assez comparables à celles rapportées par (KREUGER, 1998), pour un bassin expérimental de $9 \mathrm{~km}^{2}$, mais sur une période plus étendue.

Tableau 5 Fréquences de détection des matières actives recherchées (en \%) (cases vides : paramètres non mesurés).

Table 5 Occurrences of monitored substances, expressed as \%; empty cells correspond to non measured chemicals.

\begin{tabular}{|l|c|c|}
\hline \multicolumn{1}{|c|}{ Matière active } & Alsace & Lorraine \\
\hline Aldicarbe & 6,94 & \\
Atrazine & 56,94 & 89,94 \\
Carbendazime & 6,94 & 0,00 \\
Chlorpyriphos-éthyl & 7,64 & \\
Chlortoluron & 11,81 & 20,12 \\
Deltaméthrine & 0,00 & 0,00 \\
Diuron & 57,64 & 33,73 \\
Isoproturon & & 29,59 \\
Lambda-cyhalothrine & & 0,00 \\
Linuron & & 0,59 \\
Parathion-méthyl & 0,69 & \\
Pendiméthaline & 1,39 & \\
Prosulfocarbe & & 1,78 \\
Thiodicarbe & 2,78 & \\
Trifluraline & 2,78 & 2,96 \\
\hline
\end{tabular}

\section{4 - DISCUSSION}

La pertinence de la méthode de sélection des substances à mesurer au niveau régional dépend essentiellement de trois éléments :

- la pertinence de la méthode elle-même : en d'autres termes, a-t-on choisi les bons facteurs, ont-ils un poids approprié, etc. ? ;

- le choix des bornes, en particulier le rang d'exposition à partir duquel on considère le passage dans les eaux comme probable ; 
- la qualité des données utilisées.

Plus précisément, le troisième élément dépend :

- de l'accessibilité des données standards (caractéristiques physicochimiques, demi-vie, DJA, écotoxicité) utilisées ;

- de la fiabilité des données régionales (dose d'emploi, surface développée).

Ainsi certains composés pourront être absents des listes simplement parce que certaines données font défaut ; c'est par exemple le cas du tébutame, herbicide largement employé sur colza en Lorraine (CETIOM, 1995), mais absent du tableau de pénalités au moment où la sélection a été faite. Cela dit, on ne peut porter d'appréciation sur l'ampleur de ce défaut avec le jeu de données disponibles ici, puisque de fait les substances non sélectionnées n'ont pas été recherchées dans le cadre du RNB.

La pertinence du choix des facteurs, ainsi que du choix de la borne, s'évaluent en relation avec le rang d'exposition. Ces éléments vont pouvoir s'apprécier à partir des résultats obtenus, singulièrement des fréquences de détection ; les autres descripteurs possibles - concentrations maximales, indicateurs de la distribution des concentrations notamment - dépendent en effet largement de facteurs non pris en compte dans la démarche de sélection, comme les aléas météorologiques en relation avec les dates d'application des produits (ZHANG et al., 1997 ; RITTER, JOHNSON et al., 1974 ; LEONARD et al., 1979 ; THURMAN et al., 1991). Même sur des bassins versants de taille beaucoup plus réduite, il s'avère difficile d'établir des relations quantitatives satisfaisantes entre des facteurs explicatifs et les concentrations ou les flux exportés : ainsi par exemple (KREUGER et TÖRNQVIST, 1998), sur un bassin de $9 \mathrm{~km}^{2}$ seulement, identifient les quantités appliquées comme le plus important des facteurs responsables des quantités transportées dans la rivière, le second étant le coefficient de partage octanol-eau (Kow). Selon les années, les coefficients de corrélation $\left(R^{2}\right)$ varient de 0,54 à 0,83 à l'exutoire de ce petit bassin versant expérimental.

La relation entre la fréquence de détection et le rang d'exposition est d'allure exponentielle (figure 1), avec cependant d'une part des substances dont la fréquence de détection est nulle, jusqu'à un rang d'exposition de l'ordre de 50 , et d'autre part des substances - parfois dotées de rangs d'exposition inférieurs aux précédentes - dont les fréquences de détection paraissent diverger de ce qu'on attendrait. Ces différences doivent pouvoir s'expliquer soit par les données de classification employées, qui entraîneraient une erreur d'estimation du rang d'exposition, soit par des particularités locales, accroissant les transferts dans certains bassins versants. Les anomalies concernent les composés aldicarbe, chlorpyrifos-éthyl, carbendazime et chlortoluron.

\subsection{Aldicarbe et chlorpyrifos-éthyl}

Ces composés sont dotés de rangs d'exposition (respectivement 38 et 36) proches de la limite retenue pour le transfert vers les eaux superficielles (35). Leurs fréquences de détection (tableau 5) sont cependant nettement supérieures à celles de composés dotés de rangs d'exposition plus élevés, par exemple la pendiméthaline (Direction régionale de l'Agriculture d'Alsace et 


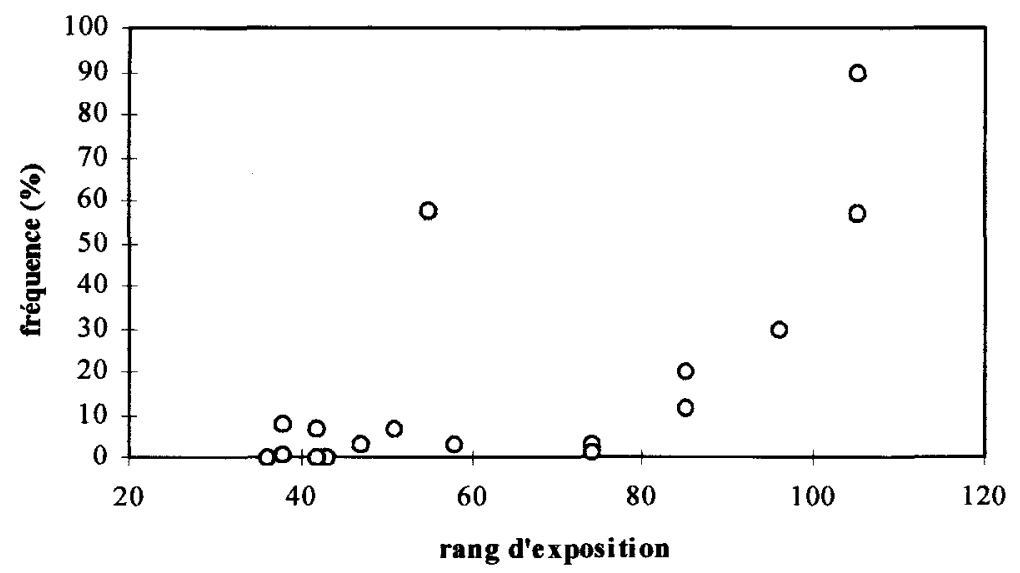

Figure 1 Fréquences de détection en fonction du rang d'exposition.

Occurences versus exposure rank.

Agricole ), rang 74 et fréquence $\approx 1,4 \%$, la trifluraline (Direction régionale de l'Agriculture de Lorraine et Agricole ), rang 58 et fréquence $\approx 3 \%$. Ces divergences donnent à penser soit que les données d'entrée sont surestimées pour les composés moins fréquemment détectés, ou sous-estimés pour ceux qui le sont plus, soit que le poids de certains critères (c'est-à-dire leur place dans l'ordre des pénalités) est mal apprécié. On remarque par ailleurs que les détections d'aldicarbe viennent de 5 points de prélèvement situés dans les parties centrale et septentrionale de l'Alsace, à l'exception de l'Ill à Tagolsheim (tableau 6). La situation est analogue pour le chlorpyrifos-éthyl.

Tableau 6 Présence d'Aldicarbe, Chlorpyrifos-éthyl et Carbendazime dans les eaux superficielles alsaciennes (nombre de détections/nombre de mesures $>$ limite de dosage dans la région).

Table 6 Detection of Aldicarb, Chlorpyrifos-ethyl and Carbendazime in Alsacian rivers (ratio number of detections/number of measures $>$ quantification limit).

\begin{tabular}{|c|c|c|c|c|}
\hline Rivière & Nom du point & Aldicarbe & Chlorpyrifos-E. & Carbendazime \\
\hline III & Tagolsheim & $3 / 10$ & & \\
Andlau & Fegersheim & & $1 / 9$ & $3 / 9$ \\
Bruche & Holtzheim & $1 / 10$ & & $1 / 9$ \\
Souffel & Mundolsheim & $3 / 10$ & $3 / 9$ & $2 / 9$ \\
Zorn & Bietlenheim & & $2 / 9$ & \\
Moder & Auenheim & $1 / 10$ & $2 / 9$ & $1 / 9$ \\
Sauer & Beinheim & & & $2 / 9$ \\
Seltzbach & Niederroedern & $2 / 10$ & $1 / 9$ & \\
\hline
\end{tabular}




\subsection{Carbendazime}

Ce composé est doté du même rang d'exposition (47) dans les deux régions ; pourtant, il n'est détecté que dans des rivières alsaciennes (tableau 5). En pratique, ces détections concernent 5 points de mesure, soit autant de bassins versants, sur les 11 surveillés dans cette région. Ces points de mesure sont situés dans la partie centrale et septentrionale de la région (tableau 6) ; les détections sont rapportées entre juillet et octobre. Ces différents éléments orientent vers une origine liée à la culture du chou, bien qu'il ne représente qu'environ $0,3 \%$ de l'assolement (SAU) dans le département du Bas-Rhin (Direction régionale de l'Agriculture d'Alsace). Toutefois, l'absence de détections en Lorraine pourrait être un artéfact : dans cette région, l'emploi du carbendazime est lié essentiellement à la culture du colza, qui représente 10,5 à $10,8 \%$ de la surface agricole utile (SAU) en 1996 et 1997 (Direction régionale de l'Agriculture de Lorraine). L'absence de détection dans cette région devrait donc moins être liée à une classification erronée qu'à une pluviométrie déficiente sur la période d'observation ; il s'avère en effet, d'après les données pluviométriques de 3 stations réparties selon un transect Nord-Sud, que les mois de mars et d'avril sont déficitaires en Lorraine, par rapport à la moyenne des 7 années précédentes.

\subsection{Chlortoluron}

Ce composé est également doté du même rang d'exposition (85) dans les deux régions ; pourtant, la fréquence de détection (tableau 5) est environ deux fois plus importante en Lorraine qu'en Alsace. Dans les deux régions, ces détections se répartissent sur l'ensemble des points de mesures, même si certains ressortent pour 1/3 environ du nombre total des détections. En l'état actuel des données, il paraît difficile de déterminer l'origine de cette différence ; il pourrait s'agir soit de différences de nature des sols, non prises en compte dans le classement préalable puisqu'on a utilisé les mêmes valeurs de Koc, soit d'une erreur d'estimation des surfaces développées, qui n'ont pas été déterminées de la même manière dans les deux cas, soit encore, comme dans le cas du carbendazime, des conséquences d'aléas climatiques.

Les divergences citées, à l'exception toutefois du chlortoluron, doivent aussi être mises en relation avec la taille du jeu de données. En effet, le nombre de résultats de mesures entrant dans le calcul des fréquences est réduit, et un écart d'une seule mesure modifie donc fortement la fréquence observée. Les anomalies apparentes peuvent donc aussi traduire une certaine sur-représentation des points de prélèvement du centre et du nord de l'Alsace ; elles ne pourront être pleinement appréciées que lorsque plusieurs années de mesures seront disponibles, et que les surfaces développées seront connues avec plus de précision qu'au moment de l'élaboration des listes de substances à surveiller.

Si l'on écarte les composés dotés de rangs d'exposition inférieurs à 50 , pour ne pas conserver de fréquences nulles dans le jeu de données, et que l'on ne prend pas en compte le diuron, qui n'a pas d'usage agricole en Lorraine et relève d'usages mixtes en Alsace, il devient possible d'ajuster les résultats à une courbe exponentielle, d'équation :

$$
y=0,0235 * e^{0.0739 x}\left(r^{2}=0,821\right)
$$

où $x$ représente le rang d'exposition (figure 2). 


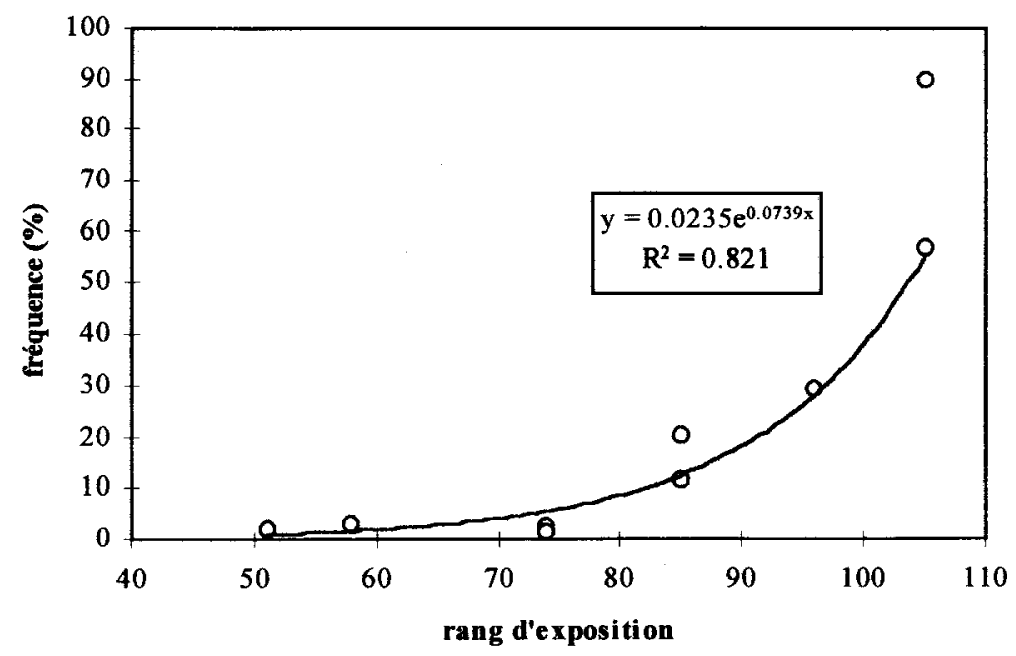

Figure 2 Relation entre fréquence de détection et rang d'exposition (substances avec rang $>50$ ).

Relation ship between occurences and exposure ranks (for substances with rank > 50).

L'introduction de méthodes d'analyses dites " multirésidus " est réputée permettre de s'affranchir de ce besoin de sélectionner les matières actives à rechercher (IFEN, 1998). Ces méthodes combinent des mélanges de solvant et des détecteurs peu ou non spécifiques (BARCELO, 1993), et ne sont donc dans leur principe pas très différentes de celles qui ont été utilisées ici, où l'on a également analysé des groupes de composés (tableau 3). En revanche, le protocole d'extraction appliqué dans le cas des méthodes " multirésidus " permet effectivement d'élargir la gamme des substances mesurées. Cet avantage paraît cependant d'une portée limitée, notamment en ce qui concerne les molécules figurant sur les listes et qui n'ont pu être analysées en routine, en raison de leur mauvaise extractabilité. Ce problème peut parfois être contourné par des approches spécifiques, comme la dérivatisation, qui améliore le rendement d'extraction ou la séparation chromatographique, ou encore la détection (cas des méthyl-carbamates, tableau 3). Les méthodes " multirésidus ", qui obtiennent un élargissement de la gamme des composés extraits par le choix de conditions moyennes d'extraction et de détecteurs non spécifiques, ne sont de ce fait pas en mesure d'apporter d'avantage déterminant pour ces composés peu extractibles.

\section{5 - CONCLUSIONS ET PERSPECTIVES}

Le rang d'exposition 50 correspond donc à la valeur au-dessus de laquelle la présence dans les eaux superficielles paraît systématique. Cependant, le rang de 35 retenu arbitrairement dans la méthode de classification originale ne 

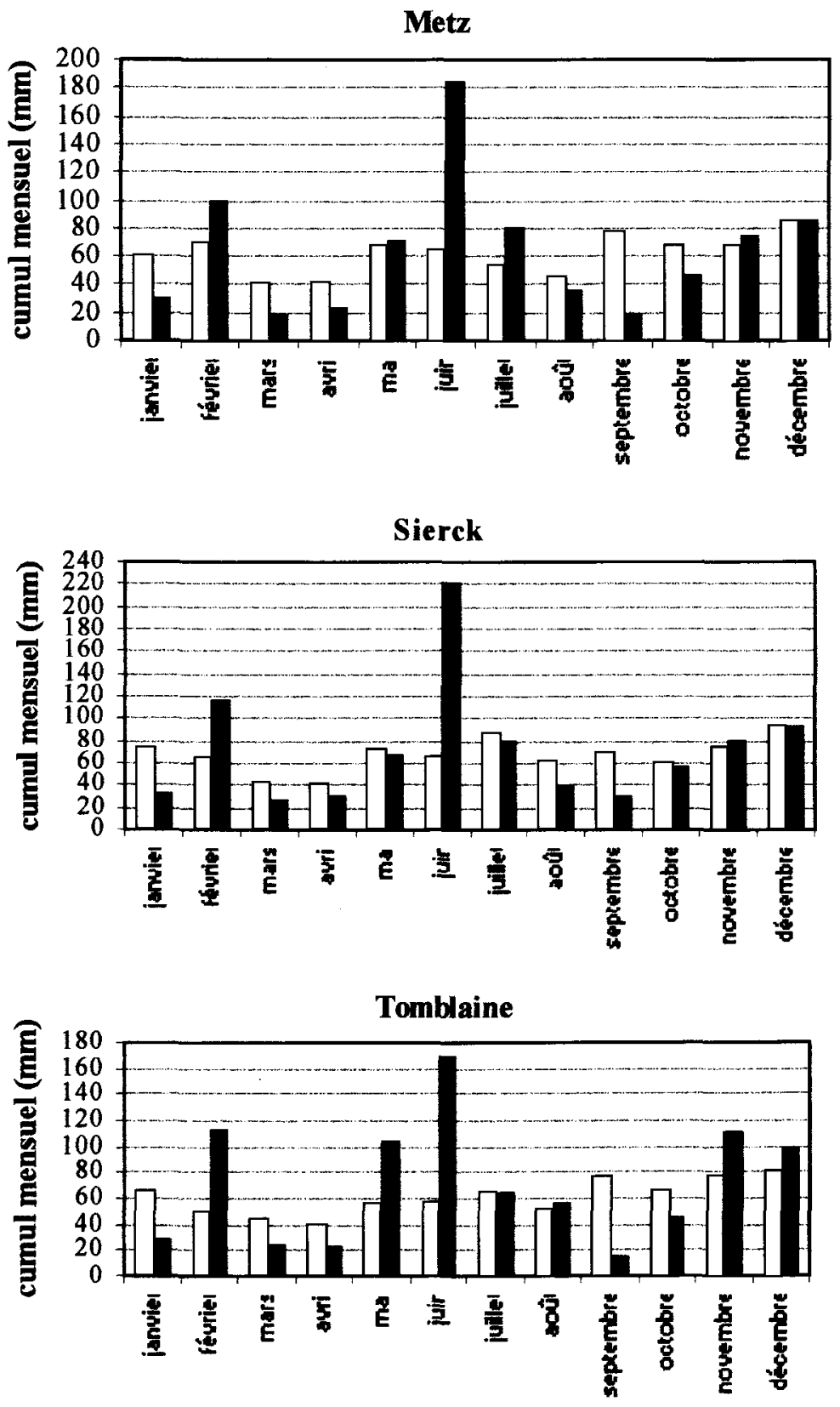

$\square$ moyenne

1997

Figure 3 Pluviométrie mensuelle moyenne et en 1997 pour 3 stations météorologiques lorraines.

Mean precipitations in Lorraine ( 3 sampling points) compared to 1977 's monthly precipitations. 
doit pas pour l'instant être modifié, puisque certains composés dotés de rangs intermédiaires sont détectés, sans que l'on puisse actuellement déterminer si leur rang d'exposition est correctement calculé ou s'il doit être ajusté. Des données supplémentaires - sur plusieurs années de mesures, et sur d'autres régions - devraient permettre de statuer sur ce point, en lissant en particulier les variations de pluviométrie.

La sélection des composés à usage agricole à surveiller dans les eaux superficielles par la méthode simplifiée d'évaluation des risques dite «SIRIS » paraît donc une démarche pertinente à l'échelle des deux régions.

\section{RÉFÉRENCES BIBLIOGRAPHIQUES}

BARCELO D., 1993. Environmental Protection Agency and other methods for the detrmination of priority pesticides and their transformation products in water. Journal of Chromatography, 643, 117143.

BEEK M. A., BROSELISKE G.H., 1994. Selection of prior pesticides and biocides within the framework of the Rhine Action Program, Haskoning Consult., RIZA. Ministère des Transports, PaysBas.

BRITT J.K., DWINELL S.E., MCDOWELL T.C., 1992. Matrix decision procedure to assess new pesticides based on relative groundwater leaching potential and chronic toxicity. Envir. Toxicol. Chem., 11, 721-728.

CETIOM, 1995. Résultats de la campagne 1994-95, régions Alsace, Lorraine et Haute-Marne. Laxou (54), CETIOM, Bureau régional de développement.

Comité de liaison « eau-produits antiparasitaires $», 1995$. Classement des matières actives phytosanitaires en vue de la surveillance de la qualité des eaux - Fonctionnement de la méthode et recommendations pour une application régionale, ministère de l'Environnement, ministère de l'Agriculture et de la Pêche, ministère des Affaires sociales, de la santé et de la ville.

Direction Régionale de l'Agriculture d'Alsace et SRdS Agricoles. Annuaire des statistiques Agricoles, années 1996 et 1997.

Direction Régionale de l'Agriculture de Lorraine and SRdS Agricoles. Annuaire des statistiques Agricoles, années 1996 et 1997.

HORNSBY A.G., 1992. Site-specific pesticide recommendations: the final step in environmental impact prevention. Weed Technology, 6, 736-742.

IFEN, 1998. Les pesticides dans les eaux collecte et traitement des données.

JOUANY J.M., DABENE E., 1994. Classement des matières actives phytosanitaires en vue de la surveillance de la qualité des eaux à l'échelle nationale, ministère de l'Environnement, ministère de l'Agriculture et de la Pêche, ministère des Affaires sociales, de la santé et de la ville, Comité de liaison « eau-produits antiparasitaires ".

JURY W., FOCHT D.D., FARMER W.J., 1987. Evaluation of pesticide pollution potential from standard indices of soil-chemical adsorption and biodegradation. J. Envir. Qual., 16, 422-428.

KREUGER, J., 1998. Pesticides in stream water within an agricultural catchment in southern Sweden, 1990-1996. Sci. Tot. Environ., 216, 227-251.

KREUGER J., TÖRNQVIST L., 1998. Multiple regression analysis of pesticide occurrence in streamflow related to pesticide properties and quantities applied. Chemosphere, 37, 189-207.

LEONARD R.A., LANGDALE G.W., FLEMING W.G., 1979. Herbicide runoff from upland piedmont watersheds - data and implications for modeling pesticide transport. J. Environ. Qual., 8, 223-229. 
NEWMAN A., 1995. Ranking pesticides by environmental impact. Environ. Sci Technol., 29, 324A-326A.

RAO P.S.C., HORNSBY A.C., JESSUP R.E., 1985. Indices for ranking the potential for pesticide contamination of Groundwater. Soil \& Crop Science Society of Florida, Proceeding.

RITTER W.F., JOHNSON H.P., LOVELY W.G., MOLNAU M., 1974. Atrazine, Propachlor, and diazinon residues in small agricultural watersheds. Environ. Sci. Technol., 8, 38-42.

SIEE et SRPV Midi-Pyrénées, 1997. La mesure des micropolluants dans le cadre du Réseau national de bassin méthodologie et recommandations Étude interagences $n^{\circ} 54$.

THURMAN E.M., GOOLSBY D.A., MEYER M.T., KOLPIN D.W., 1991. Herbicides in surface waters of the Midwestern United States: the effect of spring flush. Environ. Sci. Technol., 25, 1794-1796.

UN/ECE, 1996. Guidelines on water-quality monitoring and assessment of transboundary rivers.

ZHANG X.C., NORTON L.D., HICKMAN M., 1997. Rain pattern and soil moisture content effects on Atrazine and Metolachlor losses in runoff. J. Environ. Qual., 26, 1539-1547. 